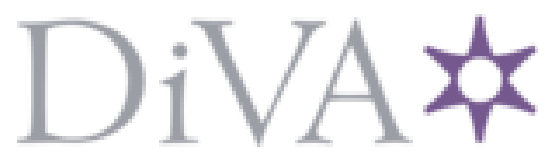

http://www.diva-portal.org

This is the published version of a paper published in Journal of Chemical Physics.

Citation for the original published paper (version of record):

Andersson, O., Johari, G P. (2016)

Thermal conductivity of Glycerol's liquid, glass, and crystal states, glass-liquid-glass transition, and crystallization at high pressures.

Journal of Chemical Physics, 144: 064504

http://dx.doi.org/10.1063/1.4941335

Access to the published version may require subscription.

N.B. When citing this work, cite the original published paper.

Permanent link to this version:

http://urn.kb.se/resolve?urn=urn:nbn:se:umu:diva-117362 


\title{
Thermal conductivity of Glycerol's liquid, glass, and crystal states, glass-liquid-glass transition, and crystallization at high pressures
}

\author{
Ove Andersson ${ }^{1}$ and G. P. Johari ${ }^{2, a)}$ \\ ${ }^{1}$ Department of Physics, Umeå University, 90187 Umeå, Sweden \\ ${ }^{2}$ Department of Materials Science and Engineering, McMaster University, Hamilton, Ontario L8S 4L7, Canada
}

(Received 3 December 2015; accepted 22 January 2016; published online 9 February 2016)

\begin{abstract}
To investigate the effects of local density fluctuations on phonon propagation in a hydrogen bonded structure, we studied the thermal conductivity $\kappa$ of the crystal, liquid, and glassy states of pure glycerol as a function of the temperature, $T$, and the pressure, $p$. We find that the following: (i) $\kappa_{\text {crystal }}$ is 3.6-times the $\kappa_{\text {liquid }}$ value at $140 \mathrm{~K}$ at $0.1 \mathrm{MPa}$ and 2.2-times at $290 \mathrm{~K}$, and it varies with $T$ according to $138 \times T^{-0.95}$; (ii) the ratio $\kappa_{\text {liquid }}(p) / \kappa_{\text {liquid }}(0.1 \mathrm{MPa})$ is $1.45 \mathrm{GPa}^{-1}$ at $280 \mathrm{~K}$, which, unexpectedly, is about the same as $\kappa_{\text {crystal }}(p) / \kappa_{\text {crystal }}(0.1 \mathrm{MPa})$ of $1.42 \mathrm{GPa}^{-1}$ at $298 \mathrm{~K}$; (iii) $\kappa_{\text {glass }}$ is relatively insensitive to $T$ but sensitive to the applied $p\left(1.38 \mathrm{GPa}^{-1}\right.$ at $\left.150 \mathrm{~K}\right)$; (iv) $\kappa_{\text {glass }}-T$ plots show an enhanced, pressure-dependent peak-like feature, which is due to the glass to liquid transition on heating; (v) continuous heating cold-crystallizes ultraviscous glycerol under pressure, at a higher $T$ when $p$ is high; and (vi) glycerol formed by cooling at a high $p$ and then measured at a low $p$ has a significantly higher $\kappa$ than the glass formed by cooling at a low $p$. On heating at a fixed low $p$, its $\kappa$ decreases before its glass-liquid transition range at that $p$ is reached. We attribute this effect to thermally assisted loss of the configurational and vibrational instabilities of a glass formed at high $p$ and recovered at low $p$, which is different from the usual glass-aging effect. While the heat capacity, entropy, and volume of glycerol crystal are less than those for its glass and liquid, $\kappa_{\text {crystal }}$ of glycerol, like its elastic modulus and refractive index, is higher. We discuss these findings in terms of the role of fluctuations in local density and structure, and the relations between $\kappa$ and the thermodynamic quantities. () 2016 AIP Publishing LLC. [http://dx.doi.org/10.1063/1.4941335]
\end{abstract}

\section{INTRODUCTION}

Thermal conductivity is a distinguishing feature of a liquid and solid, and its value for a given state varies with the density, temperature, and pressure. Crystal polymorphs of a material have different thermal conductivities, as do the glassy and other disordered states of different densities formed by different methods, or subjected to different protocols of temperature, $T$, and/or pressure, $p \cdot{ }^{1-5}$ For example, thermal conductivity, $\kappa$, is high for diamond and comparatively low for polycrystalline graphite, ${ }^{6}$ whose density is less than half of that of diamond. Also, $\kappa$ differs for polymorphs of the same density. For small crystals of cubic ice containing stacking faults of hexagonal ice structure, $k$ is $\sim 20 \%$ less than that of hexagonal ice, even though it has the same density as cubic ice. $^{7}$

Eucken ${ }^{8}$ noted that the temperature dependence of $\kappa$ of glasses, $\kappa_{\text {glass }}$, is opposite to that of crystals, $\kappa_{\text {crystal }}$. Generally speaking, $\kappa_{\text {glass }}$ increases on heating $\left(\mathrm{d} \kappa_{\text {glass }} / \mathrm{d} T>0\right)$, but $\kappa_{\text {crystal }}$ decreases $\left(\mathrm{d} \kappa_{\text {crystal }} / \mathrm{d} T<0\right)$, and the rate of change itself decreases as $T$ is increased, within the constraint of a constant $d \ln \kappa / d \ln T$ value, i.e., $\kappa$ varies as $T^{-x}$, with $x$ being a constant close to 1 . In the Debye theory for crystalline solids, $\kappa$ is determined by propagation of phonons in the crystal, and it is accepted that $\kappa_{\text {glass }}$ and $\kappa$ of other disordered solids are also determined by phonon propagation. Kittel, ${ }^{9}$ who

\footnotetext{
a)joharig@mcmaster.ca
}

theoretically discussed the magnitude and $T$ variation of $\kappa_{\text {glass }}$, argued that the phonon concepts and the Debye equation are applicable to amorphous solids and glasses. But Cahill and Pohl ${ }^{10}$ concluded that the concept of associating phonons with wave packets that travel several lattice distances in the structure of a material is useful for amorphous materials at low temperatures, but not at high temperatures when the distance was taken to decrease toward the interatomic distance. Instead of the Debye theory, they ${ }^{10}$ used the Einstein theory with an implication that the term phonon may be unsuitable for interpreting the high temperature data. Still, it has been found that the Debye equation provides a qualitative description of the variation of $\kappa_{\text {glass }}$ with $T .{ }^{9} \mathrm{Kittel}^{9}$ noted that at a given $T$, the spread of $\kappa$ values for different glasses is much less than the corresponding spread found for different crystals, and $\kappa_{\text {glass }}$ is in general lower than $\kappa_{\text {crystal. }}$. It was later found that $\kappa$ of some orientationally disordered crystals, ${ }^{1-3}$ of the pressurecollapsed state of ice, ${ }^{4,5}$ of clathrate hydrates, ${ }^{11}$ of other inclusion compounds, ${ }^{12-14}$ of filled skutterudites, ${ }^{15}$ and of some phases of normal polycrystalline materials, ${ }^{16,17}$ behaves as if they were glasses, i.e., their $\kappa$ increases on increase in $T$, or remains almost constant.

Thermal conductivity of a liquid, $\kappa_{\text {liquid, }}$, does not follow the equation for $\kappa_{\text {crystal }}$ and $\kappa_{\text {glass }}$. One expects that $\kappa_{\text {liquid }}$ in the ultraviscous range would vary as the contribution to thermodynamic properties from both phonons and the changing configurations, resulting from local density and structure fluctuations, varies. These fluctuations are observed 
as $\alpha$-relaxation in ultraviscous liquids, and in orientationally disordered crystals, and the rate of the $\alpha$-relaxation decreases as $T$ is decreased or $p$ is increased. It is also known that as the volume, $V$, and configurational entropy, $S_{c o n f}$, of a liquid increases on heating, the vibrational frequencies decrease and the effects from anharmonic forces increase. Thus, states of high configurational contributions also have a higher vibrational contribution. In as much as a change in $T$ and $p$ affects $V$ and $S_{\text {conf }}$, it would also affect its phonon properties and would thus affect $\kappa_{\text {liquid. }}$. In contrast, change in $T$ and $p$ of a glass has no effect on its $S_{\text {conf }}$. So, $\kappa_{\text {glass }}$ would vary only with change in the phonon properties with change in $V$. Hence, one expects that the equations that relate $\kappa_{\text {liquid }}$ to $T$ and $p$ would differ from those for $\kappa_{\text {glass. }}$. This difference was noted in several studies, ${ }^{18-20}$ which showed that the temperature derivative of $\kappa$ changes sign when a glass is heated through its $T_{g}$ range.

There have been numerous studies of $K$ of pure liquids and their binary mixtures at low viscosities at $0.1 \mathrm{MPa}$, the conditions in which the local density and structure fluctuations occur at nanoseconds time scale. It was found that as a liquid is cooled, $\kappa_{\text {liquid }}$ increases monotonically, i.e., $\mathrm{d} \kappa_{\text {liquid }} / \mathrm{d} T$ is negative. ${ }^{21}$ This includes inter-molecularly hydrogen bonded liquids, with water and glycerol at $298 \mathrm{~K}$ being notable exceptions. ${ }^{22,23}$ In the configurationally frozen or glassy state, in contrast, $\kappa$ decreases with decrease in $T$, i.e., $\mathrm{d} \kappa_{\text {glass }} / \mathrm{d} T$ is positive, which is interpreted in terms of the Debye equation for crystals,

$$
\kappa_{\text {crystal }}=\rho C v^{2} \tau_{s},
$$

where $\rho$ is the density, $C$ is the specific heat contribution from phonons, $v$ is the phonon propagation velocity, and $\tau_{s}$ is the time between two (phonon) scattering events. It has been suggested that the mean free path $\left(=v \tau_{s}\right)$ in the crystal varies with $T$ differently from that in the glassy state. On heating an ultraviscous liquid from $T \geq T_{g}$, the usual increase in $V$ and in $S_{\text {conf }}$ affects heat conduction such that $\mathrm{d} \kappa_{\text {liquid }} / \mathrm{d} T$ becomes negative. The change from positive value of $\mathrm{d} \kappa_{\text {glass }} / \mathrm{d} T$ to negative value of $\mathrm{d} \kappa_{\text {liquid }} / \mathrm{d} T$, or slope inversion of $\kappa-T$ plots, has been observed on heating molecular glasses and liquids, viz., glycerol, ${ }^{18,19,24}$ in the thermal diffusivity of a $\mathrm{Ge}_{20}-\mathrm{Te}_{80}$ alloy, ${ }^{25}$ polymers, ${ }^{20}$ viz., poly(methylmethacrylate), high-impact poly(styrene), normal poly(styrene), and poly(carbonate), ${ }^{26}$ poly(isoprene). ${ }^{27}$ A similar change has also been observed for orientationally disordered crystals, viz., cyclohexanol ${ }^{2}$ and cyclooctanol. ${ }^{3}$ Increase in $p$ at a fixed $T$ decreases $V$ and $S_{\text {conf }}$ and increases $T_{g}$. One expects to observe its effects on the features of $\kappa$ in the glass-liquid-glass transition range, and a shift of this range to higher $T$. Sandberg et al. ${ }^{19}$ described the use of $\kappa$ for determining $T_{g}$ at different $p$ by using glycerol as an example and also reported limited data on its $\kappa_{\text {crystal }}$. By measuring $\kappa$, Andersson and coworkers have studied the change in $T_{g}$ with $p$ of several polymers, ${ }^{27,28}$ orientational glasses, ${ }^{2,3}$ and lubricants. ${ }^{29}$

Here, we report a study (i) of the effects of $p$ and $T$ on $\kappa$ of the crystalline, glassy, and ultraviscous states of a material with an extensively hydrogen-bonded structure, (ii) the difference between $\kappa-T$ behaviors of a liquid and its crystal and the effect of applied pressure on this difference, and (iii) the effects of pressure and of thermal cycling on crystallization. We also report a corresponding study of $\rho C_{p}$ measured simultaneously with $\kappa$, where $C_{p}$ is the specific heat at a fixed $p$, and then qualitatively relate the features of $\rho C_{p}$ to those of $\kappa$.

Variation of $\kappa$ of liquids and crystals with pressure and its interpretation in terms of phonon propagation are obviously of academic interest. They are also of technological interest in tribology, especially when liquids are used as lubricants and now also as a heat dissipation source in miniaturized devices. Variation of $\kappa$ with $p$ is either ignored or indirectly estimated by combining the isothermal compressibility data with the averaged data on change in $\kappa$ with $V$ for liquids at a fixed $T$. Therefore, our study and determining $\kappa$ and $(d \kappa / d p)_{T}$ as a function of $T$ and $p$ would help initiate efforts in direct measurement of the effects of $p$ on $\kappa$ for use in tribology.

We chose glycerol for the study for the following reasons: (i) Its three $\mathrm{OH}$ groups per molecule produce a $T$ and $p$ dependent network-type hydrogen bonded structure. (ii) It shows an asymmetric distribution of relaxation times. (iii) It is reputed to be stable against crystallization but easily crystallizes under the influence of pressure. ${ }^{30}$ (iv) It is used in numerous commercial and medical products, ${ }^{31-33}$ as well as a cryoprotectant liquid-more recently as cryopreservant of biological materials. Moreover, it is beginning to be used as an environment-friendly lubricant in mechanical devices in which frictional heat generation on pressure-rise of up to GPa over a wide $T$ range and heat dissipation by conduction and phase transition are currently modeled. ${ }^{34,35}$

\section{EXPERIMENTAL METHODS}

Liquid glycerol, puriss. p.a., ACS reagent, anhydrous, dist., of $99.9 \%$ GC purity, with the main impurity being $0.07 \%$ $\mathrm{H}_{2} \mathrm{O}$ (Karl Fischer), was purchased from Sigma-Aldrich and it was studied without further purification. The transient hotwire method was used to measure the thermal conductivity $\kappa$ and the heat capacity per unit volume $\rho C_{p}$ (density times the specific heat capacity, per gm), with estimated inaccuracies of $\pm 2 \%$ in $\kappa$ and $\pm 5 \%$ in $\rho C_{p} .{ }^{36,37}$ The hot-wire probe was a semi-circular Ni-wire which was inserted in a custom-made $15 \mathrm{~mm}$ deep and $38 \mathrm{~mm}$ internal diameter Teflon ${ }^{\circledR}$ sample cell. The cell was filled with liquid glycerol and sealed with a tightly fitting Teflon lid. It was inserted in the tightly fitting cylinder of a piston-cylinder type apparatus of $45 \mathrm{~mm}$ internal diameter. The whole assembly was thereafter transferred to a hydraulic press, which supplied the load. Temperature was varied by heating or cooling the whole vessel by using a resistive heater and a built-in refrigerator, which uses a closed helium gas cycle. ${ }^{38}$ The vacuum needed for its operation eliminates frost formation, which reduces the friction between the piston and vessel. Pressure was determined from the ratio, load/area, and it was corrected for the friction. This correction was determined in a separate in situ experiment using the pressure dependence of the resistance of a manganin wire. The temperature was measured by a chromel-alumel thermocouple, which had been calibrated to within $\pm 0.2 \mathrm{~K}$ against a commercially available silicon diode thermometer. 
The "hot-wire" was subjected to a $1.4 \mathrm{~s}$ duration heatpulse of nominally constant power, and its electrical resistance was measured as a function of time. The wire acted as both the heater and the sensor for the temperature rise, which was calculated by using the relation between its resistance and temperature. The analytical solution for the temperature rise with time was fitted to the data points for the hotwire temperature rise with $\kappa$ and $\rho C_{p}$ as fitting parameters. The method is well-suited to establish the glass transition behaviour under pressure as the quantity $\rho C_{p}$ shows the typical sigmoid-shaped increase of the heat capacity $C_{p}$ and the temperature dependence of $\kappa$ often changes only weakly at the glass transition. In addition, two artificial features, namely, a peak in $\kappa$ and a dip in $\rho C_{p}$, arise. These are due to the neglected time-dependence in $C_{p}$, or imaginary part of complex heat capacity, in the glass transition region, as known from previous studies. ${ }^{39}$ The features appear in the $T$ or $p$ range over which the characteristic time of a relaxation process is of order of the probe time, i.e., about $1 \mathrm{s.}^{40,41}$ The features appear in the glass-liquid-glass range and are superimposed on the real sigmoid-shaped variations in $C_{p}$ and $\kappa$ in the $T_{g}$ and in the $P_{g}$ range.

Moreover, when the experimental conditions are near a phase boundary, e.g., the melting line, the measured values also become erroneous because of the effect of the endothermic transition on heating. ${ }^{42}$ The heating, cooling, pressurization, and depressurization rates were varied in the experiments and these rates are given where relevant in the text. Also, to search for different effects, we used a variety of experimental protocols. We include the results and interpretation. These would be useful to others in their work on the properties of glycerol and its use.

\section{RESULTS}

To investigate the $T$ and $p$ dependencies of $\kappa$ and $\rho C_{p}$, and their features in the glass-liquid-glass transition and in the cold crystallization ranges, we obtained data for the glycerol samples on both the cooling and the heating paths of a thermal cycle covering several ranges of $T$ and $p$. Figures 1 and 2 show the general pattern of changes observed in $\kappa$ as the temperature of the samples of different thermal histories was varied at a fixed $p$. In the first set of experiments, liquid glycerol under a pressure of $0.05 \mathrm{GPa}$ was cooled to $110 \mathrm{~K}$ and subsequently heated to $298 \mathrm{~K}$ while its $\kappa$ was measured continuously with changing $T$. (For brevity, its plot is not shown here.) We found that it crystallized on heating to $298 \mathrm{~K}$. The pressure was then decreased to $\sim 0.1 \mathrm{MPa}$, and the crystals were kept for $2.1 \mathrm{~h}$ at $\sim 298 \mathrm{~K}$ in order to obtain its crystal-free melt. (The equilibrium freezing point $T_{m}$ of glycerol is $291.0 \mathrm{~K}$ at ambient $(0.1 \mathrm{MPa})$ pressure, ${ }^{43}$ and $\mathrm{d} T_{m} / \mathrm{d} p$ is $66.5 \mathrm{~K} / \mathrm{GPa}^{30}$ ) It was repressurized to $0.1 \mathrm{GPa}$ and cooled under $0.1 \mathrm{GPa}$ pressure while its $\kappa$ was measured continuously. Curve 1 in Fig. 1(A) (blue upright triangles) was obtained during cooling of liquid glycerol at $0.1 \mathrm{GPa}$ from $\sim 298 \mathrm{~K}$ to $110 \mathrm{~K}$ at initially $0.5 \mathrm{~K} / \mathrm{min}$ rate, and then the cooling rate gradually decreased to $0.4 \mathrm{~K} / \mathrm{min}$ at $T$ near $210 \mathrm{~K}$, and to $0.2 \mathrm{~K} / \mathrm{min}$ at $120 \mathrm{~K}$. The curve shows a small broad peak with a maximum at $215 \mathrm{~K}$ and then a sharp local

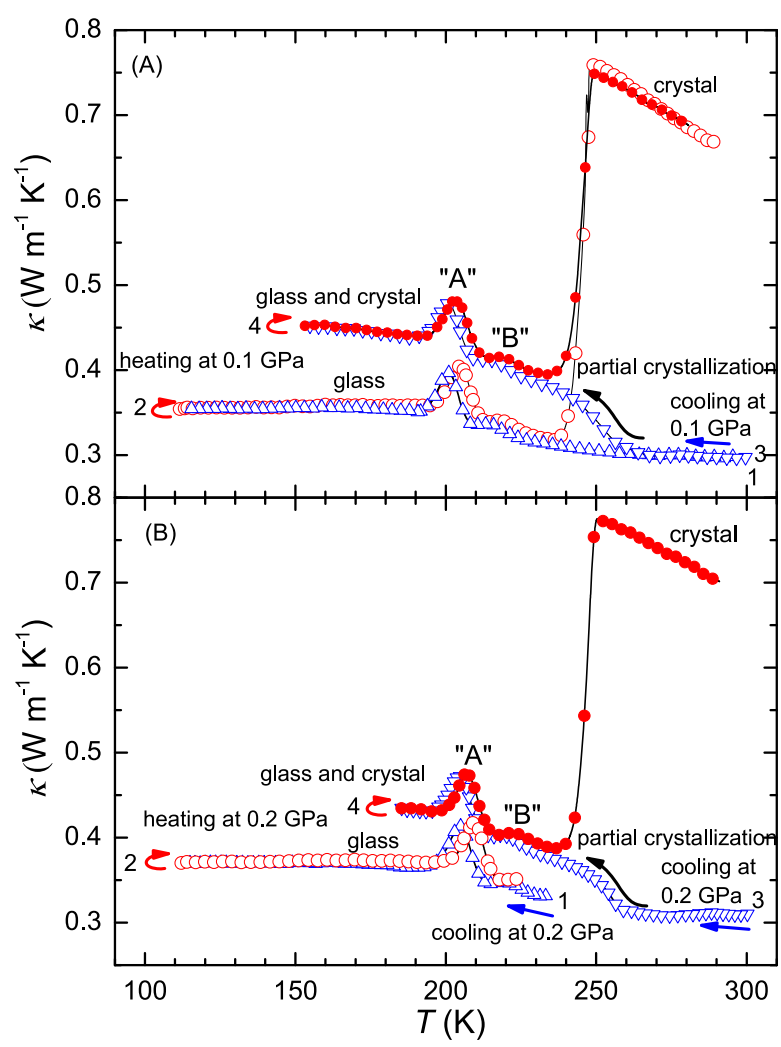

FIG. 1. Plots of $\kappa$ of liquid, glass, and crystalline states of glycerol against $T$ at two pressures obtained during the heating and the cooling paths. (A) Curve (1) is for the liquid at $0.1 \mathrm{GPa}$ obtained during cooling from $298 \mathrm{~K}$ to $110 \mathrm{~K}$ at initially $0.5 \mathrm{~K} / \mathrm{min}$ rate which gradually decreased to $0.2 \mathrm{~K} / \mathrm{min}$ (blue triangles). Curve (2) is for the subsequent heating of the glass formed to $298 \mathrm{~K}$ at initially $0.3 \mathrm{~K} / \mathrm{min}$ rate to $0.1 \mathrm{~K} / \mathrm{min}$ rate near $250 \mathrm{~K}$ at $0.1 \mathrm{GPa}$ when it crystallized (red circles). Curve (3) is for the liquid at $0.1 \mathrm{GPa}$ obtained during cooling at 0.6 to $0.4 \mathrm{~K} / \mathrm{min}$ rate (blue triangles). Curve (4) is for the subsequent heating of the glass formed to $280 \mathrm{~K}$ at 0.2 to $0.1 \mathrm{~K} / \mathrm{min}$ rate at $0.1 \mathrm{GPa}$ (red dots). (B) Curve (1) is for the liquid at $0.2 \mathrm{GPa}$ obtained during cooling from 233 to $110 \mathrm{~K}$ at an average rate of $0.4 \mathrm{~K} / \mathrm{min}$ (blue triangles). Curve (2) is for the subsequent heating of the glass formed to $230 \mathrm{~K}$ at an average rate of $0.4 \mathrm{~K} / \mathrm{min}$ at $0.2 \mathrm{GPa}$. Curve (3) is for the liquid at $0.2 \mathrm{GPa}$ obtained during cooling at an average of $0.6 \mathrm{~K} / \mathrm{min}$ to $182 \mathrm{~K}$ (blue triangles). Curve (4) is for the subsequent heating of the glass formed to $288 \mathrm{~K}$ at 0.2 $\mathrm{GPa}$ at initially $0.2 \mathrm{~K} / \mathrm{min}$ rate which decreased to $0.1 \mathrm{~K} / \mathrm{min}$ at $270 \mathrm{~K}$. Labels $\mathrm{A}$ and $\mathrm{B}$ indicate two artificial peaks in $\kappa \cdot{ }^{41}$ Instead of the peak, there is a real change in slope on configurational unfreezing of a glass on the time scale of the cooling or heating rate of the sample vessel, as discussed in the text and illustrated in the inset of Fig. 2. Further details are given in Sec. III.

peak with a maximum at $201 \mathrm{~K}$. The sample at $0.1 \mathrm{GPa}$ was subsequently heated from $110 \mathrm{~K}$ to $298 \mathrm{~K}$ at $0.3 \mathrm{~K} / \mathrm{min}$ rate, which decreased to $0.1 \mathrm{~K} / \mathrm{min}$ rate near $250 \mathrm{~K}$. The results obtained are plotted as curve 2 (red circles). It shows again the local peak, but now shifted to $205 \mathrm{~K}, 4 \mathrm{~K}$ higher than in curve 1 . On further heating, $\kappa$ shows a small, second peak, with a maximum at $218 \mathrm{~K}$, and thereafter a local minimum and then a steep rise in $\kappa$ to $0.761 \mathrm{~W} \mathrm{~m}^{-1} \mathrm{~K}^{-1}$ at $250 \mathrm{~K}$ and finally an almost linear decrease in $\kappa$ to $0.668 \mathrm{~W} \mathrm{~m}^{-1} \mathrm{~K}^{-1}$ at $290 \mathrm{~K}$. The steep rise is due to rapid crystallization of glycerol, which began at $237 \mathrm{~K}$ and was complete at $250 \mathrm{~K}$.

For repeat experiment, we obtained glycerol liquid by keeping the initially crystalline sample at $\sim 298 \mathrm{~K}$ at $\sim 0.1 \mathrm{MPa}$ pressure for $1.1 \mathrm{~h}$. Its ultimately reached $\kappa$ value was found to be close to the $\kappa$ value of all glycerol liquid samples measured at $298 \mathrm{~K}$. To study its glass formation and crystallization 


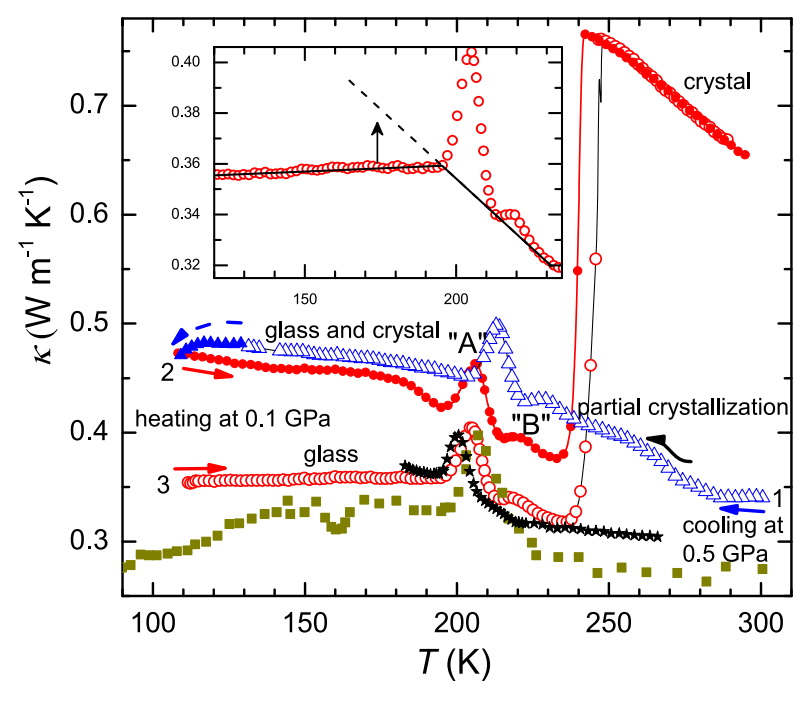

FIG. 2. The plots of $\kappa$ of liquid, glass, and crystallized samples of glycerol against the temperature at different conditions. The red color data (open and filled circles) are for heating and the blue (triangles) for cooling, with the paths indicated by the arrows. Curve (1) is for the liquid at $0.5 \mathrm{GPa}$ during cooling from $300 \mathrm{~K}$ to 130 at initially $0.7 \mathrm{~K} / \mathrm{min}$ rate, which decreased to $0.3 \mathrm{~K} / \mathrm{min}$ at $130 \mathrm{~K}$ (blue triangles). The pressure was gradually decreased from $0.5 \mathrm{GPa}$ to $0.1 \mathrm{GPa}$ during cooling from $130 \mathrm{~K}$ to $110 \mathrm{~K}$ (blue filled triangles). Curve (2) is for the glassy sample at $0.1 \mathrm{GPa}$ subsequently heated to $298 \mathrm{~K}$ at initially $0.3 \mathrm{~K} / \mathrm{min}$, which decreased gradually to $0.1 \mathrm{~K} / \mathrm{min}$ at $250 \mathrm{~K}$ (red dots). Curve (3) is for the glass at $0.1 \mathrm{GPa}$ obtained during heating at initially $0.3 \mathrm{~K} / \mathrm{min}$ rate (red circles), which decreased gradually to 0.1 $\mathrm{K} / \mathrm{min}$ at $250 \mathrm{~K}$. Labels A and B indicate two artificial peaks in $\kappa .^{41}$ Previous results at $0.1 \mathrm{MPa}$ : Cahill and Poh ${ }^{18}$ (dark yellow squares) and Birge ${ }^{39}$ (black stars). The black line in the inset illustrates the real changes in $\kappa$ on heating through the $T_{g}$ range. The slope changes when a glass configurational unfreezes kinetically on the time scale of heating and configurational freezes kinetically on the time scale of cooling, ${ }^{39,40}$ as discussed in the text. The vertical arrow illustrates the direction of change in $\kappa$ as isothermal relaxation increases the density toward the equilibrium liquid state assuming for simplicity linear extrapolation of $\kappa$ of the supercooled liquid state. Further details are given in Sec. III.

features, it was pressurized to $0.1 \mathrm{GPa}$ at $298 \mathrm{~K}$, and then cooled under $p$ of $0.1 \mathrm{GPa}$ from $298 \mathrm{~K}$ to $150 \mathrm{~K}$. The $\kappa$ measured is plotted as curve 3 (upside down blue triangles) in Fig. 1(A). During cooling at an average rate of $0.55 \mathrm{~K} / \mathrm{min}$ over the $298-150 \mathrm{~K}$ range, $\kappa$ increases in a manner different from that in curve 1 . This difference is attributed to partial crystallization on cooling from $265 \mathrm{~K}$ to $238 \mathrm{~K}$, a range in which rapid increase in the viscosity of the remaining liquid prevented full crystallization. Nevertheless, the $\kappa$ - peak still appears at the same $T$ as that of the crystals-free sample. On subsequent heating from $150 \mathrm{~K}$, the $\kappa$-peak again appears in curve 4 (solid red circles), but it is shifted slightly to higher $T$ and the small peak labeled B also appears. Thereafter, $K$ increases rapidly as crystallization continues as in curve 2 , beginning at $\sim 235 \mathrm{~K}$.

Figure 1(B) shows a similar study performed at a fixed $p$ of $0.2 \mathrm{GPa}$. Here, the $T$-range differs from that of the $0.1 \mathrm{GPa}$ study described in Fig. 1(A), but it covers the range over which the main features are evident. To obtain crystal-free glassy state, liquid glycerol was initially pressurized from $0.1 \mathrm{MPa}$ to $0.1 \mathrm{GPa}$ at $\sim 298 \mathrm{~K}$, cooled to $110 \mathrm{~K}$, reheated to $228 \mathrm{~K}$, then pressurized to $0.2 \mathrm{GPa}$ while the temperature slowly increased to $233 \mathrm{~K}$ at $0.2 \mathrm{GPa}$. (Subsequent experiments showed that completely melted glycerol crystallized on cooling from $\sim 298 \mathrm{~K}$ only at $p \geq 0.5 \mathrm{GPa}$, and therefore this procedure for obtaining crystal-free glycerol glass was unnecessary as long as $p$ remained $\leq 0.3 \mathrm{GPa}$. Nevertheless, we studied it at $p$ up to $0.5 \mathrm{GPa}$.) Ultraviscous glycerol was then cooled from $233 \mathrm{~K}$ to $110 \mathrm{~K}$ and immediately thereafter heated to $230 \mathrm{~K}$. Curve 1 (blue upright triangles) in Fig. 1(B) is for the data obtained on the cooling path and curve 2 (red circles) is for those obtained on the heating path. For a further study, liquid glycerol was obtained by keeping its crystalline sample at $\sim 298 \mathrm{~K}$ for $\sim 1 \mathrm{~h}$ at nominally $0.1 \mathrm{MPa}$, and then pressurizing the liquid to $0.2 \mathrm{GPa}$. Curve 3 (upside down blue triangles) is for the subsequent cooling path from $298 \mathrm{~K}$ and curve 4 (solid red circles) for the heating path, both at $0.2 \mathrm{GPa}$.

Figure 2 shows the features observed on the cooling path of a completely melted sample at $0.5 \mathrm{GPa}$. In these experiments, liquid glycerol, which had been kept at $298 \mathrm{~K}$ and ambient pressure $(0.1 \mathrm{MPa})$ for 3 days, was pressurized to $0.5 \mathrm{GPa}$ and then the liquid still at $0.5 \mathrm{GPa}$ was cooled isobarically initially at $0.7 \mathrm{~K} / \mathrm{min}$ rate, and this rate decreased to $0.3 \mathrm{~K} / \mathrm{min}$ at $\sim 130 \mathrm{~K}$ (curve 1 , blue upright triangles). During cooling from $130 \mathrm{~K}$ to $110 \mathrm{~K}$, the pressure was gradually decreased from $0.5 \mathrm{GPa}$ to $0.1 \mathrm{GPa}$ (curve 1 , solid blue triangles). Since the results obtained on cooling at 0.5 GPa (curve 1) are similar to those obtained on cooling a sample with some remaining nuclei at $0.1 \mathrm{GPa}$, it seems that despite lack of crystalline nuclei, partial crystallization could not be prevented on cooling at an initial rate of $0.7 \mathrm{~K} / \mathrm{min}$ at $0.5 \mathrm{GPa}$. It shows that on pressurization, the crystallization rate increases. On further cooling, $\kappa$ shows a peak, ${ }^{41}$ with a pressure induced increase in the peak temperature compared to that at $0.1 \mathrm{GPa}$, and the sample vitrified. The glassy state was then depressurized to $0.1 \mathrm{GPa}$ and immediately heated to $298 \mathrm{~K}$ at initially $0.3 \mathrm{~K} / \mathrm{min}$, and its $\kappa$ is plotted as curve 2 (solid red circles). At about $180 \mathrm{~K}$, the plot shows a slight change in the slope before peak A appears at $206 \mathrm{~K}$ and peak $\mathrm{B}$ at $221 \mathrm{~K}$, and crystallization begins at $234 \mathrm{~K}$ and ends at $244 \mathrm{~K}$ after which $\kappa$ of the crystal decreases on heating. The results for the fully vitrified (crystal-free) sample at $0.1 \mathrm{GPa}$ are plotted for comparison and shown as curve 3 (red circles, see curve 2 in Fig. 1(A) for description). It shows the peaks A and $\mathrm{B}$ and also that despite the very different thermal histories, the results of the crystal phase agree to within $0.2 \%$. The slope inversion of the $\kappa-T$ plot at $T=T_{g}$, is overwhelmed by the artificial peak ${ }^{41}$ in $\kappa$ in the glass transition range. To visualize it, we show the slope inversion as a black solid line in the inset of Fig. 2. This feature was first reported as a general behavior for amorphous polymers such as silicon rubber, polyisobutylene, polyvinylchloride, polymethyl methacrylate, and polypropylene. ${ }^{20}$ Van Krevelen used the Debye equation to reproduce the feature, and later Ross et al. ${ }^{1}$ deduced that the rise in thermal expansivity, and the associated increase in specific volume, was the main cause for the slope inversion. ${ }^{44}$ This suggests that it occurs at the vitrification temperature, which is determined by the relatively slow cooling or heating of the sample vessel. For glycerol, Cahill and Poh ${ }^{18}$ also observed a small peak at a $T$ slightly above that of slope inversion, but Birge ${ }^{39}$ found only the peak, which has been observed for other materials. ${ }^{2,3,27-29}$ Venkateshan and Johari ${ }^{45}$ observed it during the course of isothermal vitrification of 
a polymerizing mixture with time. The peak temperature is higher than the slope inversion temperature at $T=T_{g}$, and the difference increases when the probe time scale is decreased. It would change with the heating and cooling and pressurizing and depressurizing rates and the polymerizing rate, ${ }^{45}$ and it would show hysteresis on the cooling and heating and pressurizing and depressurizing paths, as evident here.

To study the $p$-dependence of $\kappa_{\text {liquid }}$ and $\kappa_{\text {crystal }}$ of glycerol, we kept one liquid sample at $\sim 0.1 \mathrm{MPa}$ at $\sim 298 \mathrm{~K}$ for $10 \mathrm{~h}$ and the second sample for $2.5 \mathrm{~h}$, and then pressurized both to $0.5 \mathrm{GPa}$ at $0.2 \mathrm{GPa} / \mathrm{h}$ rate. Figure $3(\mathrm{~A})$ shows the results on pressurizing the first sample (circles) and then depressurizing to $\sim 0.1 \mathrm{MPa}$ at the same rate. On depressurizing, the original $\kappa$ value was recovered after $\sim 1 \mathrm{~h}$ at $0.1 \mathrm{MPa}$ at $298 \mathrm{~K}$. The data plotted as squares in Fig. 3(A) (second sample) show the reproducibility of the $\kappa$ values. No data were obtained on depressurizing this sample.
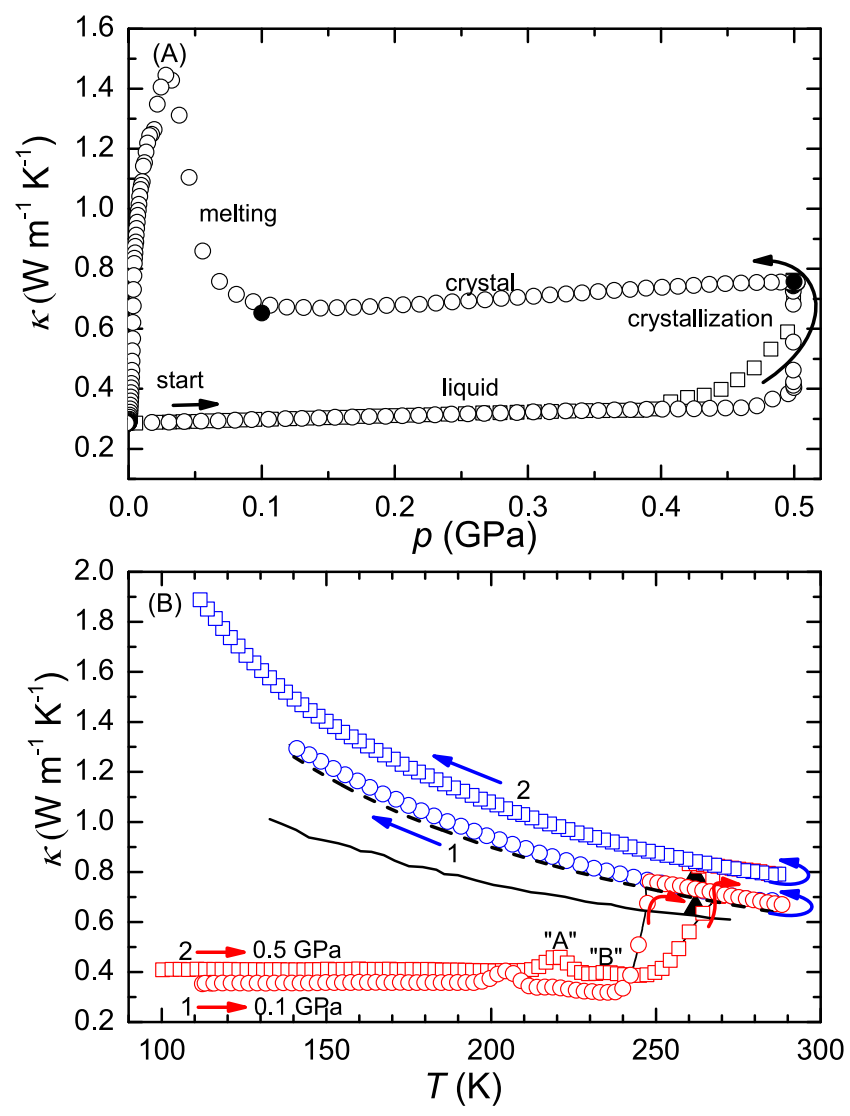

FIG. 3. (A) Plots of $\kappa$ of the liquid, glass, and crystalline glycerol at about $298 \mathrm{~K}$ as a function of pressure (black circles) first with pressurizing rate of $0.2 \mathrm{GPa} / \mathrm{h}$ rate up to $0.5 \mathrm{GPa}$ and then with decreasing pressure at $0.2 \mathrm{GPa} / \mathrm{h}$ rate after keeping for $1.3 \mathrm{~h}$ at $0.5 \mathrm{GPa}$. Data from a second experiment at the same pressurization rate of $0.2 \mathrm{GPa} / \mathrm{h}$ are plotted as black squares. The two black dots are the values obtained from isobaric measurements at 0.1 and 0.5 $\mathrm{GPa}$, shown in (B), and extrapolated to $298 \mathrm{~K}$. The data on depressurizing at $p<0.1 \mathrm{GPa}$ show an increase in the measured value, which is an artefact of the measurement procedure when the crystal melts. ${ }^{42}$ (B) Plots of $\kappa$ against $T$ obtained on heating of glass, liquid, and crystalline glycerol at $0.1 \mathrm{GPa}$ (curve 1 , red circles) and at $0.5 \mathrm{GPa}$ (curve 2 , red squares), and thereafter on isobaric cooling of crystalline glycerol at the same pressures (blue symbols). The solid line and solid triangles represent, respectively, data of Sandberg et al. ${ }^{19}$ data measured at $10 \mathrm{MPa}$ and results at 0.1 and $0.5 \mathrm{GPa}$ from isothermal pressurization. The dashed line represents our high-pressure data extrapolated to $0.1 \mathrm{MPa}: \kappa_{\text {cryst }}(0.1 \mathrm{MPa})=138 \times T^{-0.95}$ in the $140-290 \mathrm{~K}$ range. Labels $\mathrm{A}$ and B indicate two artificial peaks in $\kappa .{ }^{41}$
To investigate the $T$-dependence of $\kappa$ of glassy and crystalline glycerol at a fixed $p$, we pressurized the liquid at $298 \mathrm{~K}$ from nominally $0.1 \mathrm{MPa}$ to $0.1 \mathrm{GPa}$ pressure, and then cooled it at a fixed $p$ of $0.1 \mathrm{GPa}$. This produced the glassy state. Curve 1 (red circles) in Fig. 3(B) was obtained on subsequent heating at $0.1 \mathrm{GPa}$. It shows that glycerol glass first became ultraviscous liquid and then crystallized at $240 \mathrm{~K}$ and thereafter $\kappa_{\text {crystal }}$ decreases on heating above $248 \mathrm{~K}$. The crystalline sample was then cooled and its $\kappa$ measured (blue circles). The overlap of the data on the cooling and heating paths shows that no further crystallization occurred. In a further study, glycerol glass was made by cooling the liquid under $0.1 \mathrm{GPa}$ pressure from $298 \mathrm{~K}$ to $\sim 110 \mathrm{~K}$, then heating to $T=227 \mathrm{~K}$, where it remained above its $T_{g}$ and did not crystallize. On subsequent pressurizing from $0.1 \mathrm{GPa}$ to $0.5 \mathrm{GPa}$ (with temperature cycling at fixed $p$ of $0.1 \mathrm{GPa}$ while its $T$ increased to $242 \mathrm{~K}$ ), and finally cooling at $0.5 \mathrm{GPa}$ to $100 \mathrm{~K}$, vitrified the liquid. The glass at $0.5 \mathrm{GPa}$ was then heated at an average rate of $0.15 \mathrm{~K} / \mathrm{min}$ rate to $298 \mathrm{~K}$. The $\kappa$ measured is plotted as curve 2 (red squares) in Fig. 3(B). The results are similar to those observed for the sample studied at $0.1 \mathrm{GPa}$ pressure. Crystalline glycerol was then cooled at $0.5 \mathrm{GPa}$ (blue squares). The $\kappa$ values at $0.5 \mathrm{GPa}$ obtained on the heating and the cooling paths also overlap. Equilibrium crystallization and melting of glycerol under pressure were studied from dielectric measurements previously, where its $T_{m}$ against $p$ plot was given. ${ }^{30}$

For comparison, we include the plots of isothermal data from the work of Sandberg et al. ${ }^{19}$ (solid triangles), and the data measured at $10 \mathrm{MPa}$ (line) in Fig. 3(B). At $262 \mathrm{~K}$ and $0.1 \mathrm{GPa}$, we find $\kappa=0.731 \mathrm{~W} \mathrm{~m}^{-1} \mathrm{~K}^{-1}$, which is $9 \%$ higher than $0.657 \mathrm{~W} \mathrm{~m}^{-1} \mathrm{~K}^{-1}$ determined from their plots. ${ }^{19}$ They also determined the pressure dependence in terms of the fraction $\kappa(1 \mathrm{GPa}) / \kappa(0.1 \mathrm{MPa})$ and reported 1.42 and 1.44 for the liquid phase at 243 and $298 \mathrm{~K}$, respectively, and 1.48 for the crystal phase at $278 \mathrm{~K} .{ }^{19}$ To facilitate comparison, we calculated the corresponding values for our sample: $\kappa(1 \mathrm{GPa}) / \kappa(0.1 \mathrm{MPa})=1.45$ for the crystal at $280 \mathrm{~K}$ and 1.42 for the liquid at $298 \mathrm{~K}$ and 1.38 for glassy glycerol at $150 \mathrm{~K}$. The temperature dependence of $\kappa$ is conveniently described in terms of the parameter $x$ in the function $\kappa \sim T^{-x}$, where $x$ is close to 1 as for well-ordered monoatomic crystals. Sandberg et al. ${ }^{19}$ calculated $x=0.7$, whereas we find $x=0.94$ at $0.1 \mathrm{GPa}$ and 0.93 at $0.5 \mathrm{GPa}$. Because of the significant difference and the lack of data for $\kappa$ of crystalline glycerol at $0.1 \mathrm{MPa}$, we have extrapolated our high-pressure data measured at $0.1 \mathrm{GPa}$ and $0.5 \mathrm{GPa}$ to $0.1 \mathrm{MPa}$, which gives $\kappa_{\text {cryst }}(0.1 \mathrm{MPa})=138 \times T^{-0.95}$ in the $140-290 \mathrm{~K}$ range and the data are represented by the dashed line in Fig. 3(B).

We also measured $\rho C_{p}$ simultaneously with $\kappa$, and in the same sequence of $T$ and $p$ as for the $\kappa$ plotted in Figs. 1-3. The plots of $\rho C_{p}$ against $T$ of samples of different $T$ and $p$ histories, as well as at different $p$, are shown in Fig. 4 , where the data correspond to the protocols given for curves 1-4 in Figs. 2(A) and 2(B). Thus, curve 1 in Fig. 4(A) shows the feature in $\rho C_{p}$ on cooling and complete vitrification of the sample at $0.1 \mathrm{GPa}$. Since the density generally changes only weakly with temperature, the behavior is mainly determined by the changes in the heat capacity, which decreases gradually 


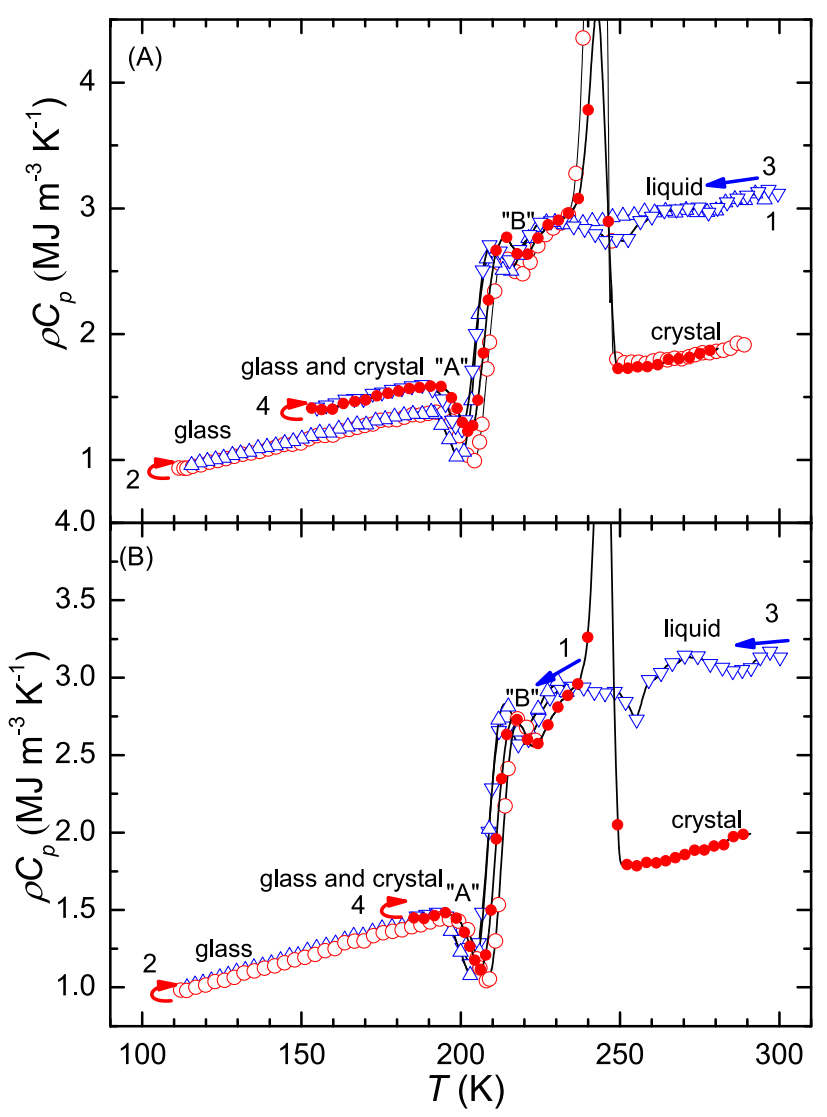

FIG. 4. Plots of $\rho C$ of glycerol liquid and glass samples of different thermal history against $T$ during heating and cooling of the glass, liquid, and the crystallized samples. (A). Curve (1) is for the liquid obtained during cooling the liquid at $0.1 \mathrm{GPa}$ from $298 \mathrm{~K}$ to $110 \mathrm{~K}$ at initially $0.5 \mathrm{~K} / \mathrm{min}$ rate which gradually decreased to $0.2 \mathrm{~K} / \mathrm{min}$ (blue triangles). Curve (2) is for the subsequent heating of the glass formed to $298 \mathrm{~K}$ at initially $0.3 \mathrm{~K} / \mathrm{min}$ rate to $0.1 \mathrm{~K} / \mathrm{min}$ rate near $250 \mathrm{~K}$ at $0.1 \mathrm{GPa}$ when it crystallized (red circles). Curve (3) is for the liquid during cooling at $0.1 \mathrm{GPa}$ at $0.6-0.4 \mathrm{~K} / \mathrm{min}$ rate (blue triangles). Curve (4) is for the subsequent heating of the glass formed to $280 \mathrm{~K}$ at $0.2-0.1 \mathrm{~K} / \mathrm{min}$ rate at $0.1 \mathrm{GPa}$ (red dots). (B). Curve (1) is for the liquid during cooling from 233 to $110 \mathrm{~K}$ at $0.2 \mathrm{GPa}$ at an average rate of $0.4 \mathrm{~K} / \mathrm{min}$ (blue triangles). Curve (2) is for the subsequent heating of the glass formed to $230 \mathrm{~K}$ at an average rate of $0.4 \mathrm{~K} / \mathrm{min}$ at $0.2 \mathrm{GPa}$. Curve (3) is for the liquid during cooling at $0.2 \mathrm{GPa}$ at an average of $0.6 \mathrm{~K} / \mathrm{min}$ to $182 \mathrm{~K}$ (blue triangles). Curve (4) is for the subsequent heating of the glass formed to $288 \mathrm{~K}$ at $0.2 \mathrm{GPa}$ at initially $0.2 \mathrm{~K} / \mathrm{min}$ rate which decreased to $0.1 \mathrm{~K} / \mathrm{min}$ at $270 \mathrm{~K}$. Labels $\mathrm{A}$ and B indicate two artificial dips in $\rho C_{p}$, as discussed in the text. Further details are given in Sec. III.

on cooling. Moreover, superimposed on the gradual decrease, the data show first a small, more abrupt, decrease before the major drop at vitrification; these occur at about the sample temperatures as the artificial, small and large peaks observed in $\kappa \cdot{ }^{41}$ Subsequent heating shows the reverse behavior but with a small temperature upshift, at least partly due to a slight increase in pressure caused by the reversed frictional forces. As the sample crystallized, $\rho C_{p}$ decreased significantly to a similar level to that of glassy glycerol. In Figs. 4(A) and 4(B), curve 3 at 0.1 and $0.2 \mathrm{GPa}$ shows that the samples partially crystallized on cooling and that $\rho C_{p}$ of the glass-crystal mixture is higher than $\rho C_{p}$ of the $100 \%$ glassy glycerol. On subsequent heating, kinetic-unfreezing occurs at slightly higher temperatures than on cooling, and on further heating the samples crystallizes completely. The data in different runs at $0.1 \mathrm{GPa}$ agree to within $1.6 \%$ after complete crystallization.
There are also small dips in $\rho C_{p}$ at the temperature of partial crystallization, i.e., near $250 \mathrm{~K}$ on cooling (curves 3 in the plots in Fig. 4). As discussed previously (see Fig. S4 in supplementary information of Ref. 46), these dips and the slightly higher $\rho C_{p}$ observed here for the mixture of glass and the crystal phase must be interpreted with caution, because mixed phases and heat evolution during crystallization can produce erroneous $\rho C_{p}$ data.

\section{DISCUSSION}

Glycerol crystallizes into an orthorhombic structure, with four symmetry-equivalent molecules per unit cell of dimensions: $a=7.00 \pm 0.04 \AA, b=9.96 \pm 0.05 \AA$, $c=6.29$ $\pm 0.04 \AA$. Positions of all carbon and oxygen atoms have been established, but not those of the hydrogen. Its structure in the liquid and crystal states has been simulated in computation ${ }^{47}$ and the extents of hydrogen bonding determined under pressure. ${ }^{48}$ By performing ab initio calculations for the positions of hydrogen atoms in the crystal structure of glycerol, it was proposed that there is a three-dimensional hydrogenbonded network of intermolecular hydrogen bonds in its crystal structure. ${ }^{49}$ In discussing our findings, we use this as background knowledge of hydrogen bonds in glycerol and of its effect on phonon propagation.

Our study reveals several aspects of the effects of phonon scattering on $\kappa$ of glycerol's crystal, liquid, and glassy states, including the aspects of transformation between these states, the configurational freezing of glycerol liquid on cooling and unfreezing on heating, and the corresponding features of the specific heat. It also reveals another effect on its $\kappa$, which results from additional instability of a glass formed by cooling at high $p$, which is gradually lost on heating at a low $p$. This type of instability was discussed in the context of $C_{p}$ and enthalpy of high density amorph of water, ${ }^{50}$ where it was suggested that a calorimetric study of glycerol glass formed at high $p$ and studied at $0.1 \mathrm{MPa}$ pressure would show similar changes in $C_{p}$ and the enthalpy. We discuss these findings in Secs. IVA-IVC.

\section{A. Thermal conductivity, configurational properties, and kinetic freezing and unfreezing of local fluctuations}

Thermal conductivity of crystals and its variation with $T$ and $p$ are interpreted in terms of the Debye theory by using Eq. (1), which is analogous to the corresponding equation in the kinetic theory of gases. The theory itself was initially based upon collective vibrational modes (phonons) with no contribution from anharmonic forces, but Debye later included these to understand the finite $\kappa$ value of crystals. In Eq. (1), $\tau_{s}$ varies as $T$ and $p$ are varied while the overall crystal structure or state does not necessarily change. This variation is mainly attributed to the change in the occupation of phonon modes and its effect on Umklapp and defect scattering events. ${ }^{51}$ Equation (1) is used also to interpret the $\kappa-T$ variation of a glass in which there is no order for molecular or atomic positions. Kittel, ${ }^{9}$ who critically reviewed the Debye equation for crystals and the $\kappa_{\text {glass }}-T$ variation, deduced that the mean free path of phonons decreases with increase in $T$ (Fig. 3 in 
Ref. 9) but to a much lesser extent than it does in crystals, and $\tau_{s}$ for glasses remains almost constant with changing $T .{ }^{9}$ After the discovery of the $\kappa_{\text {glass }}-T$ like variation of $\kappa$ of orientationally disordered crystals, it seemed that $\tau_{s}$ in such crystals also does not vary with $T$.

Bridgman $^{52}$ used a simple physical picture of "the total energy transferred across a fixed point of any row of molecules per unit time as the product of the energy difference and the number of such energy steps in a row of molecules..." in certain length. He deduced a formula relating $\kappa_{\text {liquid }}$ to its thermodynamic properties and the sound velocity $c$,

$$
\kappa_{\text {liquid }}=2.8 \mathrm{k}_{\mathrm{B}}\left(\frac{c}{V_{m}^{2 / 3}}\right) \text {, }
$$

where $\mathrm{k}_{\mathrm{B}}$ is the Boltzmann constant and $V_{m}$ is the molecular volume, i.e., the molecular mass divided by the density. According to the work of Hirschfelder, ${ }^{53}$ Eyring also gave a formula,

$$
\kappa_{\text {liquid }}=2.8 \mathrm{k}_{\mathrm{B}}\left(\frac{c}{\gamma^{1 / 2} V_{m}^{2 / 3}}\right)=2.8 \mathrm{k}_{\mathrm{B}} V_{m}^{-2 / 3}\left(\frac{1}{\rho \beta}\right)^{1 / 2},
$$

where $\gamma=C_{p} / C_{v}$, where $C_{v}$ is the heat capacity at a constant volume. According to Eqs. (1) and (2), the variation of $\kappa_{\text {liquid }}$ with $T$ and $p$ depends upon the variation of $C_{p}, C_{v}$, and $\beta$ with $T$ and $p$ through $c\left[=(\gamma / \rho \beta)^{1 / 2}\right]$, where $\beta$ is the isothermal compressibility and $\rho$ is the density.

As a liquid's $\eta$ increases on cooling through the glass formation range, $c$ increases and $V_{m}$ decreases. Both cause $\kappa_{\text {liquid }}$ to increase on cooling, i.e., $\mathrm{d} \kappa_{\text {liquid }} / \mathrm{d} T$ is negative. When the glass has formed, Eqs. (2) and (3) no longer apply, $\kappa_{\text {glass }}$ follows Eq. (1), and its value decreases on cooling, i.e., $\mathrm{d} \kappa_{\text {glass }} / \mathrm{d} T$ is positive. The opposite occurs on heating a glass through its $T_{g}$ range in which a glass softens to ultraviscous liquid, and the positive value of $\mathrm{d} \kappa_{\text {glass }} / \mathrm{d} T$ becomes the negative value of $\mathrm{d} \kappa_{\text {liquid }} / \mathrm{d} T$. Pressurizing at a fixed $T$ has an effect qualitatively similar to that of cooling, and depressurizing has an effect qualitatively similar to heating. ${ }^{27,29}$ Bridgman $^{52}$ noted that while Eq. (2) provides a description of the magnitude and of the temperature change of $\kappa_{\text {liquid }}$, it does not provide a good quantitative description of the pressure dependence of $\kappa$. Variation of $\kappa$ with $p$ reported over an otherwise wide $T$-range here is important because such data are rare and often needed for modeling of fluids behavior in tribology. Because of the difficulties in measuring $\kappa$ at high $p$, one ignores the effect of pressure on $\kappa$. Alternatively, one uses the variation of $\kappa_{\text {liquid }}$ with the density, $\rho$, of liquids at a fixed $T$, which for most liquids is empirically given by, $\kappa_{\text {liquid }} \sim \rho^{x}$, with $x$ close to $3,{ }^{1}$ and combines it with the compressibility data to obtain the variation of $\kappa_{\text {liquid }}$ with $p$. For water at ambient and higher $T$, however, $x=2.0{ }^{1}$ By using data of McDuffie et $a l .{ }^{54}$ for the variation of $\rho$ with $p$ at $303 \mathrm{~K}$, we find that $x=2.0$ also for liquid glycerol at $\sim 298 \mathrm{~K}$.

A dip and then a broad step-like increase in the $\rho C_{p}$ against $T$ plots in Fig. 4 appear in the $T$-range of the $\kappa$ peak, which are similar to the corresponding features observed also for other materials. ${ }^{2,3,19,27,29}$ As described earlier here, the artificial $\kappa$ peak (of magnitude $\sim 0.05 \mathrm{~W} \mathrm{~m}^{-1} \mathrm{~K}^{-1}$ in curve 2 and 4 , Fig. 1) and the $\operatorname{dip}$ in $\rho C_{p}$ appearing on both the cooling and heating paths are due to experimental method using the hot wire or hot strip and the analytical procedure with the neglected imaginary part of complex $C_{p}$, as explained previously, ${ }^{39-41}$ i.e., the dip is not due to a decrease in $C_{p}$. As the change in $\rho$ is negligibly small in the narrow $T_{g}$ range, the $\rho C_{p}-T$ plots in the glass transition range therefore reflect the change in $C_{p}$, and the increase in $\rho C_{p}$ after this apparent dip is due to the real $C_{p}$ increase associated with kinetic unfreezing on the time scale of the transient heating, i.e., when the heat capacity relaxation time becomes less than about $1 \mathrm{~s}$. Thus, the $\rho C_{p}-T$ plots provide information complimentary to the $\kappa-T$ plots.

In the $T$-range of $190-230 \mathrm{~K}$ of the plots obtained on cooling and heating in Figs. $1-4$, both $\kappa$ and $\rho C_{p}$ of the cooling curves show two features labeled A and B in Figs. 1(A), 2, 3(B), and 4(A). Both resemble the features observed in the glass-liquid transition range of other materials. Feature B is smaller than the feature $\mathrm{A}$ and it was found to appear at $T$ which is $\sim 13 \mathrm{~K}$ higher at $\sim 0.1 \mathrm{MPa}$ (data not shown here), and $\sim 15 \mathrm{~K}$ higher at $0.5 \mathrm{GPa}$ than feature A. Feature $\mathrm{A}$ in both $\kappa$ and $\rho C_{p}$ is a characteristic of kinetic freezing of molecular motions on cooling and unfreezing on heating. Therefore, feature B is also attributable to kinetics freezing on cooling and unfreezing on heating, i.e., there are further slower modes of molecular motions that kinetically unfreeze at $T$ that is higher than $T_{g}$. Sandberg et al. ${ }^{19}$ reached the same conclusion, but they did not search for the origin of the increase in $\rho C_{p}$. We found that the $C_{p}$-spectroscopy data by Birge (Fig. 1, Ref. 39) show a similarly weak second sigmoid shape increase in the fixed frequency measurements for glycerol at $0.1 \mathrm{MPa}$ pressure, which corresponds to feature B here. In his study, the position of this feature shifted to higher $T$ as the frequency was increased. But it was not observed in the (complimentary) dielectric relaxation spectra; the spectra showed only a low-frequency broadening. ${ }^{30,55} \mathrm{~A}$ second relaxation feature in glycerol has been observed in ultrasonic studies at $0.1 \mathrm{MPa}$ pressure at $T>270 \mathrm{~K},{ }^{56}$ but it is not certain whether or not it has the same origin as $C_{p}$. Birge also reported it in the $\kappa-T$ plots of glycerol obtained by thinwire measurements, as a kink-like feature in the $210-220 \mathrm{~K}$ range, in Fig. 7(B) in Ref. 39 but did not clearly observe it in the less accurate measurements using plane heaterthermometer. ${ }^{39}$ We also note that a second relaxation feature at high temperatures has been observed in both the $\kappa-T$ plots and dielectric spectra of poly(propylene glycol), $\mathrm{PPG},{ }^{57-59}$ poly (isobutylene), ${ }^{60}$ and polyisoprene. ${ }^{27,57,58}$ Both features $A$ and $B$ and their increased separation on increasing the pressure have been observed for PPG, ${ }^{59}$ poly (isobutylene) ${ }^{60}$ and polyisoprene. In PPG, features A and B were associated with local segmental modes ( $\alpha$-relaxation) and slower chain modes (normal modes), ${ }^{61,62}$ which were separated in dielectric and mechanical relaxation spectra, but not in $C_{p} .{ }^{63}$ The two processes became well resolved in their $\kappa-T$ and $\rho C_{p}-T$ plots at high pressure. ${ }^{59}$

As described earlier here, the artificial $\kappa$-peak is superposed on the feature of gradual slope inversion in the $\kappa$ against $T$ plot, whose temperature increases with increase in $p$. Similar $\kappa$-peaks were found in studies of linear chain and network structure polymerization occurring isothermally in real time and for the same reason as here, except that the 
$\kappa$-peak appeared at that time during polymerization when the increasing structural relaxation time of the polymerized state became comparable to the probe time. ${ }^{45}$ The difference in the peak temperatures here increases from about $13 \mathrm{~K}$ at $0.1 \mathrm{MPa}$ ( $197 \mathrm{~K}$ and $210 \mathrm{~K}$, data not shown) to $15 \mathrm{~K}$ at $0.5 \mathrm{GPa}(216 \mathrm{~K}$ and $231 \mathrm{~K}$, Fig. 3(B)). The macroscopic relaxation time, which is determined by the rapid heating rate of the probe, is the same at the temperatures of both peaks. So, the results show that $(\partial T / \partial p)$ for a fixed relaxation time for feature $\mathrm{B}$ is somewhat larger, thereby indicating a further separation of features $\mathrm{A}$ and $\mathrm{B}$ with increase in $p$.

Although data on feature B for molecular liquids are rare, there is sufficient information to discuss what its origin could be. The feature is observed on both the heating and cooling paths of $\kappa$ against $T$ plots, with slight hysteresis, and therefore it is associated with the $T$ - and time-dependent fluctuations dynamics relative to the time scale of the experiment. It has been observed in polymers, ${ }^{27,57-60}$ and in glycerol, ${ }^{39}$ in the latter case in the $C_{p}$ and, possibly also in the ultrasonic measurements, ${ }^{56}$ but not observed in dielectric relaxation spectra or fixed frequency permittivity and loss data measured as a function of $T$. Strictly interpreted, feature B would correspond to diffusive motions that produce local density and structure fluctuations that contribute to the volume and entropy but do not change the dipole vector. Hence, they appear in $C_{p}$ and the ultrasonic data but not in dielectrics. For large molecules, we envisage that parts of the molecule locally fluctuate without a change in the dipole moment. For small molecules, rotation about the $C_{2 v}$ (two fold symmetry axis as in the case of a water molecule), rotation about the dipolar vector, rotational translational motions of a non-dipolar molecules, or non-dipolar segments of a long molecule, would all contribute to the entropy and hence to $C_{p}$ but not to dielectric polarization. ${ }^{64}$ Such fluctuations in poly(propylene glycol), ${ }^{57-59}$ poly (isobutylene), ${ }^{60}$ and polyisoprene. ${ }^{27,57,58}$ may show features in the $\kappa-T$ plots when both their normal and segmental modes contribute while widely separated at the time scale of $\sim 0.3 \mathrm{~s}$ used for the $\kappa$ measurements. Their dielectric spectra already show both features A and B. There is a need to specifically look for feature $\mathrm{B}$ in well characterized liquids by the $\kappa, C_{p}$, and ultrasonic and dielectric measurements.

\section{B. The effect of pressure on crystallization}

Glycerol is known to be a liquid that does not crystallize in the impure state, but in the pure state, it was found to crystallize in a few cases. It was also found that when its crystals were melted and then the liquid was cooled, it crystallized readily. ${ }^{43}$ It also crystallized more easily under pressure. ${ }^{30,65}$ The plots in Figs. 1-3 show that glycerol only partially crystallizes on cooling and fully cold-crystallizes when either the uncrystallized or partially crystallized liquid is heated at a high pressure. Also, crystallization occurs over a broad $T$-range. On heating at $0.15 \mathrm{~K} / \mathrm{min}$, under $p$ of 0.5 GPa, crystallization begins at $247 \mathrm{~K}$ (Curve 2, Fig. 3(B)) and the onset $T$ and the $T$-range are higher for $0.5 \mathrm{GPa}$ pressure than for $0.1 \mathrm{GPa}$ with crystallization onset at $237.5 \mathrm{~K}$. These data indicate the effect of diffusion- or viscosity-control on the rate of crystallization. When crystallization occurs on heating, the viscosity, $\eta$, decreases and the crystallization rate increases and when it occurs on cooling, $\eta$ increases and the crystallization rate decreases. The partially crystallized sample has a higher $\kappa$ and greater $\mathrm{d} \kappa / \mathrm{d} T$ than the liquid alone as if $\kappa$ were additive for the liquid and crystal states.

To discuss the effect of pressure on the crystallization rate, we first note that the samples annealed for $2.5 \mathrm{~h}$ at $298 \mathrm{~K}$ at 0.1 $\mathrm{MPa}$ and thereafter isothermally pressurized at $0.2 \mathrm{GPa} / \mathrm{h}$ rate to $0.4-0.5 \mathrm{GPa}$ range (Fig. 3(A)) show qualitatively the same slow rate of crystallization as the sample at $0.1 \mathrm{MPa}$ annealed for $10 \mathrm{~h}$ at $298 \mathrm{~K}$, and then similarly pressurized. The $2.5 \mathrm{~h}$ annealed sample showed onset of crystallization at $0.4 \mathrm{GPa}$ and the $10 \mathrm{~h}$-annealed sample showed this onset at $0.47 \mathrm{GPa}$. This suggests that the effect of increased annealing for $7.5 \mathrm{~h}$ at $298 \mathrm{~K}$ at $0.1 \mathrm{MPa}$ only slightly increased the crystallization onset pressure. The results indicate that glycerol samples of the purity used here, $99.9 \%$ with less than $0.07 \%$ water, do not vitrify by pressurizing at $0.2 \mathrm{GPa} / \mathrm{h}$ or lower rate, at $\sim 298 \mathrm{~K}$.

The plots in Fig. 1(A) show that, on cooling at 0.1 $\mathrm{GPa}$, the rate of crystal growth is maximum near $252 \mathrm{~K}$, which corresponds to $T / T_{g}=0.77 \pm 0.02$, with $T_{g}=195 \mathrm{~K}$ estimated for a relaxation time of $10^{2} \mathrm{~s}$ (on heating). ${ }^{41}$ For the corresponding maximum at $271 \mathrm{~K}$ at $0.5 \mathrm{GPa}$ (Fig. 2), we find $T / T_{g}=0.78 \pm 0.02$, with $T_{g}=212 \mathrm{~K}$, which shows that this ratio does not strongly depend upon the pressure. Cook et al. ${ }^{66}$ found that on cooling, $\eta$ initially increases at a higher rate at 0.1 MPa than at high $p$, i.e., $(\mathrm{d} \eta / \mathrm{d} T)_{p=0.1 \mathrm{MPa}}>(\mathrm{d} \eta / \mathrm{d} T)_{p>0.1 \mathrm{MPa}}$, and we also deduce that $\eta$ at maximum crystal growth rate is $\sim 1 / 10$ th at $0.4 \mathrm{GPa}$ than at $0.1 \mathrm{MPa}$. If nucleation and crystal growth processes were controlled by $\eta$, the finding may indicate that both processes are more rapid at low $\eta$ than at high $\eta$. This is in conflict with the fact that glycerol at the same $T$ crystallizes at high $p$ when $\eta$ is high but not at 0.1 MPa when $\eta$ is low. It is not known whether crystallization is promoted more by increase in local density of its hydrogen bonded structure, increase in the extent of hydrogen bonding, which increases with increasing pressure, ${ }^{48}$ or by the increase in energy and decrease in the entropy caused by a high pressure.

\section{Configurational instability of high-pressure formed glass}

On heating at the same $p$ of $0.2 \mathrm{GPa}$ (curves 2 and 4, Figs. 1(A) and 1(B)), the $\kappa$-peak at the glass-liquid transition appears at the same $T$ as the $\kappa$ peak for the partially crystalline and crystal-free liquid, as if the presence of crystals has no effect on the $\kappa$ peak position. In Fig. 2, curve 1 shows that when the partially crystallized liquid at $0.5 \mathrm{GPa}$ is cooled, it vitrifies, as expected, to a partially crystallized glass. Its comparison with curves 1 and 3 in Fig. 1 shows that for about the same cooling rate, vitrification at $0.5 \mathrm{GPa}$ occurs at a higher $T$ than vitrification of the partially crystallized and crystal-free liquid cooled at $0.2 \mathrm{GPa}$, i.e., $\mathrm{d} T_{g} / d p$ is positive. There is no other feature before the onset of this peak.

In a review of the properties of glasses vitrified at high pressures and studied by differential scanning calorimetry at $0.1 \mathrm{MPa}$ pressure, one of $\mathrm{us}^{50}$ discussed an additional instability of the high pressure formed glasses in the context of a previously mistaken second $T_{g}$ of water. This instability may 
be observed under isothermal conditions at $0.1 \mathrm{MPa}$ pressure as the sample tends to stabilize towards its equilibrium state, or may be more easily observed on heating at $0.1 \mathrm{MPa}$ pressure. On the basis of previous studies, he stressed ${ }^{50}$ that $H$ and $V$ of a glass formed by cooling under a high $p$ and studied at 0.1 MPa pressure are lower than the $H$ and $V$ of the glass formed by cooling at $0.1 \mathrm{MPa}$ and studied at $0.1 \mathrm{MPa}$ pressure. ${ }^{50} \mathrm{To}$ elaborate, when a glass formed at a high $p$ is heated at a low $p$, of say $0.1 \mathrm{MPa}$, the following three processes occur before its state becomes ultraviscous liquid at $0.1 \mathrm{MPa}$ pressure. (i) $H$ and $V$ increase as the instability caused by glass formation at a high pressure is gradually lost on heating at $0.1 \mathrm{MPa}$. (ii) $H$ and $V$ decrease as the glass structure stabilizes by irreversible relaxation toward the $H$ and $V$ values of its equilibrium liquid state at $0.1 \mathrm{MPa}$. (Spontaneous decrease in $H$ and $V$ is a characteristic of all glasses studied at the same pressure, of usually $0.1 \mathrm{MPa}$, at which they were formed.) (iii) The slope of the plot of $H$ and $V$ at the onset of glass to liquid transition increases and this increase is observed as a broad sigmoidshape increase in $C_{p}$ and in $\mathrm{d} V / \mathrm{d} T$. The first two occurrences are sub- $T_{g}$ effects which would be observed for all glasses formed under high $p$ and studied by heating at a low $p$.

Curve 2 in Fig. 2 shows that when the glass formed by cooling at $0.5 \mathrm{GPa}$ is heated at a fixed pressure of $0.1 \mathrm{GPa}$, $\kappa_{\text {glass }}$ decreases on heating from $180 \mathrm{~K}$ to $195 \mathrm{~K}$, below the calorimetric $T_{g}$ of $195 \mathrm{~K}$ at $0.1 \mathrm{GPa}$. The $\kappa$-peak on heating at $0.1 \mathrm{GPa}$ appears at about the same $T$ as the $\kappa$-peak of the crystal-free sample vitrified by cooling at $0.1 \mathrm{GPa}$ and heated under $0.1 \mathrm{GPa}$ pressure. This is expected because the relaxation time is $0.3 \mathrm{~s}$ at the temperature of all $\kappa$-peaks in this study and the presence of crystal only increases the magnitude of $\kappa$. (Note that the thermal relaxation time is $\sim 100 \mathrm{~s}$ at $\sim 195 \mathrm{~K}$, which is also below the temperature of the artificial peak in $\kappa \cdot{ }^{41}$ )

It is known that $\kappa_{\text {glass }}$ generally increases as its volume decreases on pressurizing, ${ }^{1,2}$ or decreases as its volume increases on depressurizing, with the low-density amorphous ice as an exception. ${ }^{5}$ Therefore, we suggest, that the decrease observed here indicates the effects of increase in volume on stabilization of the glass structure to its true volume at 0.1 $\mathrm{GPa}$, as anticipated from a previous discussion of the glasses formed under pressure and studied at $0.1 \mathrm{MPa} .{ }^{50}$ The effect is opposite to that of physical aging which decreases the volume and would increase $k_{\text {glass }}$, as indicated by the arrow in the inset of Fig. 2, and shown by Hiki et al. ${ }^{67}$ in a study of $\kappa$ of AgI- $\mathrm{AgPO}_{3}$ during physical aging at $T<T_{g}$. Partial crystallization of the sample before vitrification on cooling at $0.5 \mathrm{GPa}$ reduced the fraction of liquid that vitrified on cooling at $0.5 \mathrm{GPa}$. This reduced the sub- $T_{g}$ effect on $\kappa$ observed on heating the sample at $0.1 \mathrm{GPa}$.

\section{CONCLUSIONS}

Both the structure and dynamics determine the thermal conductivity of glycerol crystal and liquid. Phonon scattering is determined by its crystal structure and by the dynamics of rotational translational diffusion and hydrogen-bond breaking and reforming in its liquid. Combination of the two sources produces certain features in $\kappa$ not observed in other physical properties.
When the glycerol crystals melt, the onset of rotational and translational motions decreases $\kappa$, as is generally found on melting. In the glassy state when these motions are kinetically frozen, the sign of temperature coefficient of $\kappa$ changes to a small positive value. There is a greater difference between $\kappa$ of glass and crystal phase than between $\kappa$ of the liquid and crystal phase. $\kappa$ of the crystal phase increases with increase in the pressure slightly more rapidly than of the liquid at $298 \mathrm{~K}$, while $(d \ln \kappa / d \ln T)_{p}$ at a given $T$ remains constant with changing $p$, i.e., $x$ in their respective $\kappa \propto T^{-x}$ does not change with $p . \kappa$ of the glassy state is relatively insensitive to $T$ at a fixed $p$ and sensitive to the applied pressure at a fixed $T$.

The plots of $\kappa$ against $T$ show a local peak whose position depends upon $p$. The peak is due to the glass to liquid transition on heating. On continuous heating under pressure, ultraviscous glycerol cold-crystallizes at a temperature that is higher when the pressure is higher, i.e., not only is $d T_{\text {cryst }} / d p$ positive at the (equilibrium) liquid-crystal phase boundary with $T_{\text {cryst }}$ being the temperature of crystallization, it is also positive for (non-equilibrium or irreversible) cold crystallization below the phase boundary, at lower $T$.

The glassy state of glycerol formed at a high pressure and studied at a low pressure by heating from a low temperature shows a sub- $T_{g}$ feature in which $\kappa$ decreases prior to its glass-liquid transition range is reached at the low pressure. Since glass formed by cooling under a high pressure is denser than a glass formed by cooling at a lower pressure, there would be a spontaneous decrease in the density (increase in volume) of such a glass with time at a low pressure, which would be accelerated on heating. The sub- $T_{g}$ feature is due to the spontaneous recovery of the lower density (larger volume) state corresponding to the lower pressure. The effect is analogous to the effect observed in calorimetry when a glass formed at a high pressure shows a sub- $T_{g}$ endothermic peak in $C_{p}$ when heated at $0.1 \mathrm{MPa}$ pressure before the $T$-range of glass-liquid transition at $0.1 \mathrm{MPa}$ pressure is reached ${ }^{68}$ (Also see Fig. 2 and the relevant discussion in Ref. 50.)

Our directly measured $\kappa$ and $\mathrm{d} \kappa / \mathrm{d} p$ values as a function of $p$ and $T$ provide accurate values for modeling in tribology, especially when a combination of the compressibility with an estimated $\kappa_{\text {liquid }}-V$ plots yields $\kappa_{\text {liquid }}-p$ variation with large errors from both the data used and their fitted curves. For convenience of modeling, we have provided tables of data as supplementary material in Ref. 69.

\section{ACKNOWLEDGMENTS}

This work was supported by the Swedish Research Council, Grant No. 621-2012-3336. We acknowledge financial support for equipment from Carl Trygger Foundation and Magnus Bergvalls foundation.

${ }^{1}$ R. G. Ross, P. Andersson, B. Sundqvist, and G. Bäckström, Rep. Prog. Phys. 47, 1347 (1984).

${ }^{2}$ O. Andersson, R. G. Ross, and G. Bäckström, Mol. Phys. 66, 619 (1989).

${ }^{3}$ O. Andersson and R. G. Ross, Mol. Phys. 71, 523 (1990).

${ }^{4}$ G. P. Johari and O. Andersson, J. Chem. Phys. 120, 6207 (2004).

${ }^{5}$ O. Andersson and H. Suga, Phys. Rev. B 65, 140201 (2002).

${ }^{6}$ A. A. Balandin, Nat. Mater. 10, 569 (2011).

${ }^{7}$ G. P. Johari and O. Andersson, J. Chem. Phys. 143, 054505 (2015). 
${ }^{8}$ A. Eucken, Ann. Phys. 34, 185 (1911).

${ }^{9}$ C. Kittel, Phys. Rev. 75, 972 (1949).

${ }^{10}$ D. G. Cahill and R. O. Pohl, Annu. Rev. Phys. Chem. 39, 93 (1988).

${ }^{11}$ R. G. Ross, P. Andersson, and G. Bäckström, Nature 290, 322 (1981).

${ }^{12}$ R. G. Ross, J. Inclusion. Phenom. Mol. Recognit. Chem. 8, 227 (1990).

${ }^{13}$ M. Zakrzewski and M. A. White, Phys. Rev. B 45, 2809 (1992).

${ }^{14}$ T. Takabatake, K. Suekuni, T. Nakayama, and E. Kaneshita, Rev. Mod. Phys. 86, 669 (2014).

${ }^{15}$ G. S. Nolas, D. T. Morelli, and T. M. Tritt, Annu. Rev. Mater. Sci. 29, 89 (1999).

${ }^{16}$ O. Andersson, O. Chobal, I. Rizak, V. Rizak, and V. Sabadosh, Phys. Rev. B 83, 134121 (2011).

${ }^{17} \mathrm{M}$. Tachibana, N. Taira, H. Kawaji, and E. Takayama-Muromachi, Phys. Rev. B 82, 054108 (2010).

${ }^{18}$ D. G. Cahill and R. O. Pohl, Phys. Rev. B 35, 4067 (1987)

${ }^{19}$ O. Sandberg, P. Andersson, and G. Bäckström, J. Phys. E: Sci. Instrum. 10, 474 (1977).

${ }^{20}$ D. W. Van Krevelen, Properties of Polymers (Elsevier, Amsterdam, 1972).

${ }^{21}$ D. K. H. Briggs, Ind. Eng. Chem. 49, 418 (1957).

${ }^{22}$ M. L. V. Ramires, C. A. Nieto de Castro, Y. Nagasaka, A. Nagashima, M. J. Assael, and W. A. Wakeham, J. Phys. Chem. Ref. Data 24, 1377 (1995).

${ }^{23}$ Y. S. Toulokian, P. E. Liley, and S. C. Saxena, "Thermophysical properties of matter," in Thermal Conductivity, Nonmetallic Liquids and Gases (IFI/Plenum, New York, 1970)

${ }^{24}$ A. A. Minakov, S. A. Adamovsky, and C. Schick, Thermochim. Acta 403, 89 (2003).

${ }^{25}$ Q. Xu and K. Ichikawa, J. Phys. C: Solid State Phys. 18, L985 (1985).

${ }^{26}$ W. N. dos Santos, J. A. de Sousa, and R. Gregoriom, Jr., Polym. Test. 32, 987 (2013).

${ }^{27}$ B. Tonpheng, J. Yu, and O. Andersson, Macromolecules 42, 9295 (2009).

${ }^{28} \mathrm{~J}$. Yu, B. Tonpheng, and O. Andersson, Macromolecules 43, 10512 (2010).

${ }^{29}$ R. Larsson and O. Andersson, Proc. Inst. Mech. Eng., Part J 214, 337 (2000).

${ }^{30}$ G. P. Johari and E. Whalley, Faraday Symp. Chem. Soc. 6, 23 (1972).

${ }^{31}$ J. W. Lawrie, Glycerol and the Glycols: Production, Properties and Analyses (Chemical Catalog Company, New York, 1928).

${ }^{32}$ A. R. Ubbelohde, The Molten State of Matter (Wiley, New York, 1978).

${ }^{33}$ C. S. Miner and N. N. Dalton, Glycerol (Reinhold, Baltimore, 1953).

${ }^{34} \mathrm{~S}$. Wen and P. Huang, Principles of Tribology (John Wiley \& Sons, Singapore, 2012).

${ }^{35}$ Y. Shi, I. Minami, M. Grahn, M. Björling, and R. Larsson, Tribol. Int. 69, 39 (2014).

${ }^{36}$ B. Håkansson, P. Andersson, and G. Bäckström, Rev. Sci. Instrum. 59, 2269 (1988).

${ }^{37}$ O. Andersson and A. Inaba, Phys. Chem. Chem. Phys. 7, 1441 (2005).

${ }^{38}$ O. Andersson, B. Sundqvist, and G. Bäckström, High Pressure Res. 10, 599 (1992).

${ }^{39}$ N. O. Birge, Phys. Rev. B 34, 1631 (1986).

${ }^{40}$ O. Andersson, Int. J. Thermophys. 18, 195 (1997)

${ }^{41} \mathrm{~A}$ peak in $\kappa$ and dip in $\rho C_{p}$ appear because of a time-dependence in $C_{p}$ due to the kinetic unfreezing of structural fluctuations in the glass to liquid transition range, which is not accounted for since $\kappa$ and $\rho C_{p}$ are treated as adjustable, time-independent, parameters in the fitting of the analytical solution for the temperature rise of the hot-wire. The effect is most pronounced when the $C_{p}$ change occurs during the probing time of $1.4 \mathrm{~s}$ (see experimental section). This feature has been previously analyzed in detail (Ref. 40) and it was found that the peak maximum in these experiments occurs at a relaxation time of about $0.3 \mathrm{~s}$. (It is $10^{-2} \mathrm{~s}$ at the beginning of the peak at the high $T$ end and $10^{2} \mathrm{~s}$ at the end of the peak at low $T$ end in Ref. 40.) The peak is thus superimposed on the real changes in $\kappa$ due to the temperature change and vitrification. In addition to the experimental time-scale set by the transient heating of the hot-wire probe, with an average heating rate of $150 \mathrm{~K} / \mathrm{min}(3.5 \mathrm{~K}$ in $1.4 \mathrm{~s}$ ), there is another time scale determined by the slow heating and cooling rates of the vessel of less than $1 \mathrm{~K} / \mathrm{min}$. A cooling rate of $1 \mathrm{~K} / \mathrm{min}$ in these studies typically leads to vitrification when the $\alpha$-relaxation time $\sim 100 \mathrm{~s}$, which means that the sample vitrifies at near the low- $T$ end of the low-temperature peak. (The relatively slow cooling and heating of the vessel, with a rate of less than $1 \mathrm{~K} / \mathrm{min}$, is superimposed on the rapid heating of the probe and does not cause the sample to kinetically freeze or unfreeze during the heat pulse.) The hysteresis in the $\kappa$ peak between cooling and heating paths (Figs. 1 and 2) is partly due to the reversal in the direction of friction-forces on the piston, which causes a larger pressure on heating than on cooling, and partly due to changing temperature gradients in the sample cell. At $T$ below $T_{g}$, on the left end of the $\kappa$-peak and which is determined by the cooling and heating rate, the hysteresis is partly also intrinsic due to structural freezing and unfreezing. Therefore, the peaks, which are in the 190-230 K T-range in Figs. 1(A) and 1(B), are artificial features due to neglect of the imaginary part of the complex heat capacity in data analysis of the transient hot-wire and hot-strip methods as was discussed in previous studies (Refs. 39 and 40).

${ }^{42}$ During the transient heating of the hot-wire, endothermic melting of the crystal severely reduces the temperature rise of the hot-wire in a similar manner as a very high $\kappa$ does. Thus, this effect of heat absorbed by the sample produces erroneously large values for $\kappa$. As the liquid fraction gradually increases with time, this effect decreases and $\kappa$ decreases slowly to the liquid phase value.

${ }^{43}$ G. E. Gibson and W. F. Giauque, J. Am. Chem. Soc. 45, 93 (1923).

${ }^{44}$ The slope inversion seems to be associated with the change in thermal expansion and the corresponding larger increase in volume above $T_{g}$ than below. This change should be observed when the sample kinetically unfreezes on the time scale of heating and unfreezes on the time scale of cooling. That is, the volume is larger above $T_{g}$ than below, and it changes more rapidly with $T$ at $T>T_{g}$. Change in thermal expansivity during the pulse does not cause the slope inversion, but instead the change in the initial volume and the interatomic distances. Hence, one observes two processes in hot-wire experiments, one corresponds to the relaxation time of $\sim 100 \mathrm{~s}$ of the slope inversion and the second corresponds to the relaxation time of $\sim 0.3 \mathrm{~s}$ for the peak.

${ }^{45}$ K. Venkateshan and G. P. Johari, J. Chem. Phys. 125, 054907 (2006).

${ }^{46}$ O. Andersson, Proc. Natl. Acad. Sci. U. S. A. 108, 11013 (2011).

${ }^{47}$ L. J. Root and F. H. Stillinger, J. Chem. Phys. 90, 1200 (1989).

${ }^{48}$ L. J. Root and B. J. Berne, J. Chem. Phys. 107, 4350 (1997).

${ }^{49}$ W. T. M. Mooij, B. P. van Eijck, and J. Kroon, J. Am. Chem. Soc. 122, 3500 (2000).

${ }^{50}$ G. P. Johari, Thermochim. Acta 617, 208 (2015).

${ }^{51}$ N. W. Ashcroft and N. D. Mermin, Solid State Physics (Saunders College Publishing, Philadelphia, 1976).

${ }^{52}$ P. W. Bridgman, Proc. Natl. Acad. Sci. U.S.A. 9, 341 (1923).

${ }^{53}$ J. O. Hirschfelder, C. F. Curtiss, and R. B. Bird, Molecular Theory of Gases and Liquids (John Wiley and Sons, New York, 1954).

${ }^{54}$ G. E. Mcduffie, J. W. Forbes, W. M. Madigosky, and J. J. Von Bretzel, J. Chem. Eng. Data 14, 176 (1969).

${ }^{55}$ H. Forsman, P. Andersson, and G. Bäckström, J. Chem. Soc., Faraday Trans. 2 82, 557 (1986).

${ }^{56}$ R. M. Slayton and K. A. Nelson, J. Chem. Phys. 120, 3919 (2004).

${ }^{57}$ K. L. Ngai, R. Casalini, and C. M. Roland, Macromolecules 38, 4363 (2005).

${ }^{58}$ C. M. Roland, R. Casalini, and M. Paluch, J. Polym. Sci., Part B: Polym. Phys. 42, 4313 (2004)

${ }^{59}$ S. P. Andersson and O. Andersson, Macromolecules 31, 2999 (1998).

${ }^{60}$ S. P. Andersson, J. Polym. Sci., Part B: Polym. Phys. 36, 1781 (1998).

${ }^{61}$ M. E. Baur and W. H. Stockmayer, J. Chem. Phys. 43, 4319 (1965).

${ }^{62}$ T. Alper, A. J. Barlow, and R. W. Gray, Polymer 17, 665 (1976).

${ }^{63}$ G. P. Johari, A. Hallbrucker, and E. Mayer, J. Polym. Sci., Part B: Polym. Phys. 26, 1923 (1988)

${ }^{64}$ G. P. Johari, J. Non-Cryst. Solids 307-310, 387 (2002).

${ }^{65}$ It was noted in Ref. 43 that "After the seed crystals had arrived, it was found that crystallization practically always occurred when amount of 100 $\mathrm{g}$ of any laboratory sample was slowly warmed over a period of a day, after cooling to liquid-air temperatures. This occurred even when great precautions were taken to exclude the presence of seeds. However, it was found readily possible, by temperature manipulation alone, to produce crystalline or supercooled glycerol at will." Johari and Whalley in Ref. 30 found that glycerol purified by vacuum-distillation always crystallized on cooling at high pressures, and, therefore, they used a new sample of glycerol in each of their 12-14 runs at high pressures. It was also found that a crystal of glycerol of $\sim 5 \mathrm{~mm}$ size taken out of the pressure vessel at $0.1 \mathrm{MPa}$ and kept inside a sealed bottle containing unpurified glycerol liquid, in which it sank to the bottom, did not grow perceptibly for a period of $\sim 14$ months when kept at $\sim 278$ in a refrigerator, and the liquid and crystal existed together, indicating that crystal did not grow at $0.1 \mathrm{MPa}$ pressure at $278 \mathrm{~K}$.

${ }^{66}$ R. L. Cook, H. E. King, Jr., C. A. Herbst, and D. R. Herschbach, J. Chem. Phys. 100, 5178. (1994)

${ }^{67}$ Y. Hiki, H. Takahashi, and Y. Kogure, Solid State Ionics 86-88, 463 (1996). ${ }^{68}$ I. G. Brown, R. E. Wetton, M. J. Richardson, and N. G. Savill, Polymer 19, 659 (1978)

${ }^{69}$ See supplementary material at http://dx.doi.org/10.1063/1.4941335 for tables of numerical data. 